\title{
Cruenza come violenza?
}

\author{
Ilde Consales
}

PUBBLICATO: 09 MARZO 2021

\section{Quesito:}

Alcuni lettori si sono interrogati sulla legittimità della parola cruenza come derivato dall'aggettivo cruento, dal momento che l'hanno riscontrata in pubblicazioni di varia natura, ma non risulta registrata nei dizionari.

\section{Cruenza come violenza?}

I

1 sostantivo cruenza 'sanguinosità, violenza' (dall'aggettivo cruento 'sanguinoso, che comporta spargimento di sangue') può essere considerato un caso di parola d'uso incipiente: una parola che circola in italiano, soprattutto in ambito scritto, da alcuni decenni ed è già in parte considerata accettabile dalla nostra comunità linguistica, ma che ancora non è registrata dai dizionari generali o dai repertori di neologismi, anche quelli più accurati e più ampi.

Lanciando una ricerca su internet, cruenza risulta ampiamente adoperata nella scrittura da almeno un trentennio (ma anche da prima: vedi infra) in relazione a eventi sanguinosi. Se ne trovano numerosi esempi nella prosa giornalistica (ad esempio nell'archivio storico della "Repubblica" la prima attestazione risale al 1986 :

A proposito di tifosi, quelli del Torino dopo un inizio di stagione all'insegna della cruenza, almeno verbale, si sono rilassati. (Giancarlo Emanuel, Giorno di derby ma non di caccia al biglietto..., "la Repubblica", $16 / 2 / 1986)$

e in quella saggistica:

Di questi 25 giudizi negativi, Ig si appoggiano su argomenti che fanno appello alla violenza/cruenza. (P. Bertolini, Bambini giudici della TV: rapporto di ricerca su una settimana di programmazione televisiva in fascia protetta, Milano, Guerini e associati, 2002)

Già nel Codice di Hammurabi gli imprenditori edili venivano scoraggiati (anche se in maniera indiretta e con la cruenza tipica di quel periodo storico) a servirsi di manodopera. (M. Ferdinandi, Una etica gestione delle risorse umane nei Paesi in via di sviluppo, Santarcangelo di Romagna, Fara Editore, 2006)

la cruenza e perfino l'ingenuità che accompagnarono e seppellirono i protagonisti di una delle stagioni più disperate della nostra storia (M. Merluzzi, Ad limina: percorsi storiografici di frontiera, Roma, Arance, 2008)

esposizione diretta e prolungata a forti stress, assenza di un supporto diretto, cruenza della situazione (G. Catherine Roulet, Soccorritore e vittima: Gli aspetti psicologici nello spazio relazionale, Milano, Franco Angeli, 2018).

Sul sito Scrivere. Pagine di poesia. Spazio alle emozioni, cruenza dà il titolo a una poesia di A. Vezio, pubblicata online il 27/I/2013. Il lessema torna nel verso d'apertura e non è presente per dar luogo a una rima: 
Il vento sbatte con cruenza / il bucato steso ai fili / contro il muro / e non soddisfatto strappa / dalle chiome fiorite / ogni bocciolo

Su Instagram all'hashtag \#cruenza corrispondono immagini sanguinose, come quella di una carcassa di una preda divorata da un leone.

Non producono, invece, risultati positivi ricerche lanciate in corpora in rete sull'italiano scritto (anche dei secoli passati), parlato o trasmesso: come il CORIS (COrpus di Riferimento dell'Italiano Scritto), il CODIS (COrpus Dinamico dell'Italiano Scritto), il DiaCORIS (COrpus Diacronico di Riferimento dell'Italiano Scritto), il LIS (Lessico dell'Italiano Scritto), il MIDIA (Morfologia dell'Italiano in DIAcronia), il Metamotore (Lessico dell'Italiano Scritto, Televisivo, Radiofonico). Non emergono occorrenze nemmeno dalla consultazione dell'ONLI (Osservatorio Neologico della Lingua Italiana).

Si accennava, prima, ai dizionari. La presenza di una parola nei principali vocabolari dell'uso dovrebbe in parte indicarne la diffusione fra i parlanti di una lingua e il grado di acclimatamento in una comunità linguistica. Tuttavia cruenza non è presente negli Zingarelli degli ultimi anni (compreso lo Zingarelli 202I), nel GRADIT con i suoi aggiornamenti (incluso il Supporto Digitale del 2007), nel Dardano Nuovissimo Dizionario, nel DISC. Non compare in repertori dedicati ai neologismi che testimoniano il cambiamento linguistico e sociale degli anni più recenti, come Neologismi quotidiani. Un dizionario a cavallo del Millennio di Adamo e Della Valle (2003) o in 2006 parole nuove, degli stessi autori.

Se ricercata nel portale online della Treccani, cruenza non affiora con lo statuto di lemma nelle opere lessicografiche dell'Istituto, tuttavia appare allinterno di definizioni di altre voci nei seguenti repertori: il Dizionario biografico degli italiani, vol. IX (I967) di Claudio Mutini, sotto la voce Berni, Francesco ("solo un'improvvisa immagine di morte [...] può resuscitare con la sua cruenza il senso materiale della vita") e vol. LXXXI (20I4) di Alessandro Porro sotto la voce Panzeri, Pietro ("[Panzeri] presta particolare attenzione, anche storica, all'intervento di osteotomia, pratica allora assai discussa per la sua cruenza"); il Lessico del XXI secolo (2013) sotto la voce Millar, Mark ("Le sue storie si distinguono per la cruenza di molte scene d'azione"); il Vocabolario Treccani. Neologismi. Parole nuove dai giornali (2008) sotto la voce neuroscientifico: "si espande anche alla cruenza degli stessi testi teatrali e al loro lessico fitto di similitudini dure".

Guardando al passato, cruenza non figura nelle edizioni del Vocabolario della Crusca, in dizionari storici come il GDLI, ora interrogabile in rete nel sito dell'Accademia della Crusca (né come lemma, né all'interno delle definizioni di lemmi), il TLIO o il Tommaseo-Bellini (e non è registrata in altri importanti lessici ottocenteschi, come il Dizionario Universale di Alberti di Villanova, il Tramater di Liberatore, il Giorgini-Broglio, il Fanfani, il dizionario di Cardinali, Orioli e Costa, il Dizionario della Minerva di Carrer e Federici, il Gherardini). Tuttavia in un vocabolario bilingue di epoca rinascimentale, il Dittionario italiano, et francese - Dictionnaire italien et françois di Nathanael Duez (Venezia, li Milochi, 1562), cruenza fa la sua comparsa, tradotta in francese con cruauté 'crudeltà' e preceduta da un asterisco: come il compilatore avverte nella prefazione

quanto a i vocaboli fegnati con ftellette, quello dinota, che fono parole inuecchiate, ò difufate, le quali non fi vfano più, ouero voci troppo Latine, è termini di alcun dialetto, che non è buon Francefe, né buono Italiano [...]. Ma è però necefsario di hauere i vocaboli antichi in vn Dittionario, per potere intendere alcuni buoni autori antichi.

A livello morfologico, cruenza appare come un derivato dell'aggettivo cruento. È un nome che, formatosi da un aggettivo qualificativo, esprime una qualità (con un ventaglio di sfumature: ad 
esempio la qualità in sé, "l'essere cruento", ma anche la manifestazione di una qualità in una persona, un oggetto o una certa entità) oppure la designazione di un atto (una cruenza) (cfr. Franz Rainer, Derivazione nominale deaggettivale, in Grossmann-Rainer 2004, pp. 293-313: 294-295).

Il processo di derivazione di cruenza da cruento molto probabilmente è avvenuto per analogia con violento > violenza e ricorda anche la neoformazione (cfr. Rainer, op. cit., p. 305) sanguinolento > sanguinolenza: coppie di parole semanticamente affini che presentano l'alternanza consonantica della dentale / $t$ / della base con l'affricata/ts/ del derivato. Rispetto a cruento, tuttavia, gli altri due aggettivi hanno natura diversa: terminano in -lento (e il processo sanguinolento > sanguinolenza è modellato sulla derivazione da una serie di aggettivi complessi già esistenti in italiano, come corpulento > corpulenza, fraudolento $>$ fraudolenza, opulento $>$ opulenza, sonnolento > sonnolenza). Cruento, che invece termina in -ento, in passato non ha prodotto cruenza cosi come contento non ha dato *contenza, o intento non ha dato *intenza.

La percezione di una scarsa accettabilità di cruenza ne può aver determinato l'esclusione secolare dai lemmari dei dizionari italiani, anche se oramai se ne puo ipotizzare l'accoglimento in un futuro non troppo lontano.

Nota bibliografica:

- Giovanni Adamo, Valeria Della Valle, Neologismi quotidiani. Un dizionario a cavallo del Millennio, 1998-2003, Firenze, Olschki, 2003

- Giovanni Adamo, Valeria Della Valle, 2006 parole nuove. Un dizionario di neologismi dai giornali, Milano, Sperling e Kupfer, 2006

- Francesco Alberti di Villanova, Dizionario universale critico, enciclopedico della lingua italiana, 6 voll., Lucca, Marescandoli, I797-I805.

- Francesco Cardinali, Francesco Orioli, Paolo Costa, Dizionario della lingua italiana, Bologna, Per le stampe de' Fratelli Masi e comp., I8I9-I826.

- Luigi Carrer, Fortunato Federici, Dizionario della lingua italiana, Padova, Nella Tipografia della Minerva, I827-I830,

- Giovan Battista Giorgini, Novo Vocabolario della lingua italiana secondo l'uso di Firenze, ordinato dal Ministero della pubblica istruzione ... sotto la presidenza di Emilio Broglio, Firenze, Cellini e c., I870-I 897 .

- Fabio Marri, , Parole nuove, meno nuove, troppo nuove, in "Lingua nostra", LXVII (2006), pp. IIzI22; LXVIII (2007), pp. 37-46; I07-II6; LXIX (2008), pp. 5I-60; II2-I22; LXX (2009), pp. 53-60.

- Nuovissimo Dardano: dizionario della lingua italiana, dir. da M. Dardano, Roma, Curcio, 1987.

\author{
Cita come: \\ Ilde Consales, Cruenza come violenza?, "Italiano digitale", 2020, XVI, 2021/1 (gennaio-marzo) \\ DOI: $10.35948 / 2532-9006 / 2021.5488$ \\ Copyright 2020 Accademia della Crusca \\ Pubblicato con licenza creative commons CC BY-NC-ND
}

